\title{
NORMAL TYPE Ia SUPERNOVAE FROM VIOLENT MERGERS OF WHITE DWARF BINARIES
}

\author{
R. Pakmor ${ }^{1}$, M. Kromer ${ }^{2}$, S. TAubenderger ${ }^{2}$, S. A. Sim $^{3}$, F. K. RÖPKe ${ }^{4}$, ANd W. HillebrandT ${ }^{2}$ \\ ${ }^{1}$ Heidelberger Institut für Theoretische Studien, Schloss-Wolfsbrunnenweg 35, 69118 Heidelberg, Germany \\ ${ }^{2}$ Max-Planck-Institut für Astrophysik, Karl-Schwarzschild-Str. 1, 85741 Garching, Germany \\ ${ }^{3}$ Research School of Astronomy and Astrophysics, Mount Stromlo Observatory, Cotter Road, Weston Creek, ACT 2611, Australia \\ ${ }^{4}$ Institut Für Theoretische Physik und Astrophysik, Universität Würzburg, Emil-Fischer-Str. 31, 97074 Würzburg, Germany \\ Received 2011 August 8; accepted 2012 January 23; published 2012 February 15
}

\begin{abstract}
One of the most important questions regarding the progenitor systems of Type Ia supernovae (SNe Ia) is whether mergers of two white dwarfs can lead to explosions that reproduce observations of normal events. Here we present a fully three-dimensional simulation of a violent merger of two carbon-oxygen white dwarfs with masses of $0.9 M_{\odot}$ and $1.1 M_{\odot}$ combining very high resolution and exact initial conditions. A well-tested combination of codes is used to study the system. We start with the dynamical inspiral phase and follow the subsequent thermonuclear explosion under the plausible assumption that a detonation forms in the process of merging. We then perform detailed nucleosynthesis calculations and radiative transfer simulations to predict synthetic observables from the homologously expanding supernova ejecta. We find that synthetic color light curves of our merger, which produces about $0.62 M_{\odot}$ of ${ }^{56} \mathrm{Ni}$, show good agreement with those observed for normal $\mathrm{SNe}$ Ia in all wave bands from $U$ to $K$. Line velocities in synthetic spectra around maximum light also agree well with observations. We conclude that violent mergers of massive white dwarfs can closely resemble normal SNe Ia. Therefore, depending on the number of such massive systems available these mergers may contribute at least a small fraction to the observed population of normal SNe Ia.
\end{abstract}

Key words: binaries: close - hydrodynamics - radiative transfer - supernovae: general

Online-only material: color figures

\section{INTRODUCTION}

Despite many years of dedicated research, the progenitor systems and explosion mechanisms of Type Ia supernovae (SNe Ia) remain unclear. It is generally accepted that $\mathrm{SNe}$ Ia originate from thermonuclear explosions of massive carbon-oxygen white dwarfs in binary systems. Depending on the nature of the companion star, two different progenitor systems have been proposed. In the single degenerate scenario (Whelan \& Iben 1973), a carbon-oxygen white dwarf accretes from a non-degenerate companion star until it reaches the Chandrasekhar mass and explodes (but note that an explosion before reaching the Chandrasekhar mass may also be possible, e.g., Fink et al. 2007, 2010). In contrast, in the double degenerate scenario (Iben \& Tutukov 1984; Webbink 1984) a merger of two carbon-oxygen white dwarfs causes a thermonuclear explosion of the merged system.

Many recent findings, including SN rates from population synthesis studies (Ruiter et al. 2009), studies of the delay time distribution of observed SNe Ia (e.g., Maoz et al. 2010), the lack of radio emission of SNe Ia (e.g., Hancock et al. 2011), the lack of hydrogen emission in nebular spectra of SNe Ia (Leonard 2007), the lack of X-ray emission in elliptical galaxies (Gilfanov \& Bogdán 2010), and studies of SN Ia remnants (e.g., Badenes et al. 2007) seem to favor the double degenerate scenario. In addition, first studies of SN 2011fe seem to disfavor a single degenerate progenitor (see, e.g., Bloom et al. 2012). There is, however, no unambiguous proof for any progenitor scenario yet. For a detailed discussion about constraints on the progenitor scenarios see Howell (2011).

To date, most theoretical work on SNe Ia has concentrated on the single degenerate scenario, as mergers of white dwarfs were thought not to lead to thermonuclear explosions. This was mainly based on the picture that mergers would leave behind the more massive white dwarf with a hot envelope made up of the material of the less massive white dwarf. Fast accretion from the envelope will then turn the carbon-oxygen white dwarf into an oxygen-neon white dwarf (Nomoto \& Iben 1985; Saio \& Nomoto 1998) which collapses to a neutron star as it approaches the Chandrasekhar mass (Nomoto \& Kondo 1991). Ways to avoid the transformation of the carbon-oxygen white dwarf into an oxygen-neon white dwarf have been proposed (e.g., Yoon et al. 2007), but without conclusive results.

Recently, however, we demonstrated that violent mergers of two carbon-oxygen white dwarfs could directly lead to a thermonuclear explosion while the merger is still ongoing (Pakmor et al. 2010). We also showed that the observables for such an explosion with two white dwarfs of $0.9 M_{\odot}$ show good agreement with the observed properties of subluminous $1991 \mathrm{bg}$ like SNe Ia. Furthermore, we found that for a primary mass of $0.9 M_{\odot}$ a mass ratio of at least about 0.8 is required for the scenario to work (Pakmor et al. 2011).

Recently, Dan et al. (2011) showed that using exact initial conditions can change the properties of the merger. In particular, this leads to a significantly longer inspiral in their simulations. However, Dan et al. (2011) were only able to run the simulation with a resolution of $2 \times 10^{5}$ particles (for comparison, the violent merger calculations by Pakmor et al. 2010, 2011 used $2 \times$ $10^{6}$ particles).

In this Letter, we combine high-resolution merger simulations with exact initial conditions. We present the results of a simulation of the massive merger of a $1.1 M_{\odot}$ and a $0.9 M_{\odot}$ carbon-oxygen white dwarf. We follow the evolution of the binary system through the merger phase, thermonuclear explosion, and nucleosynthesis. Finally, we use three-dimensional radiative transfer simulations to obtain synthetic light curves and spectra. 


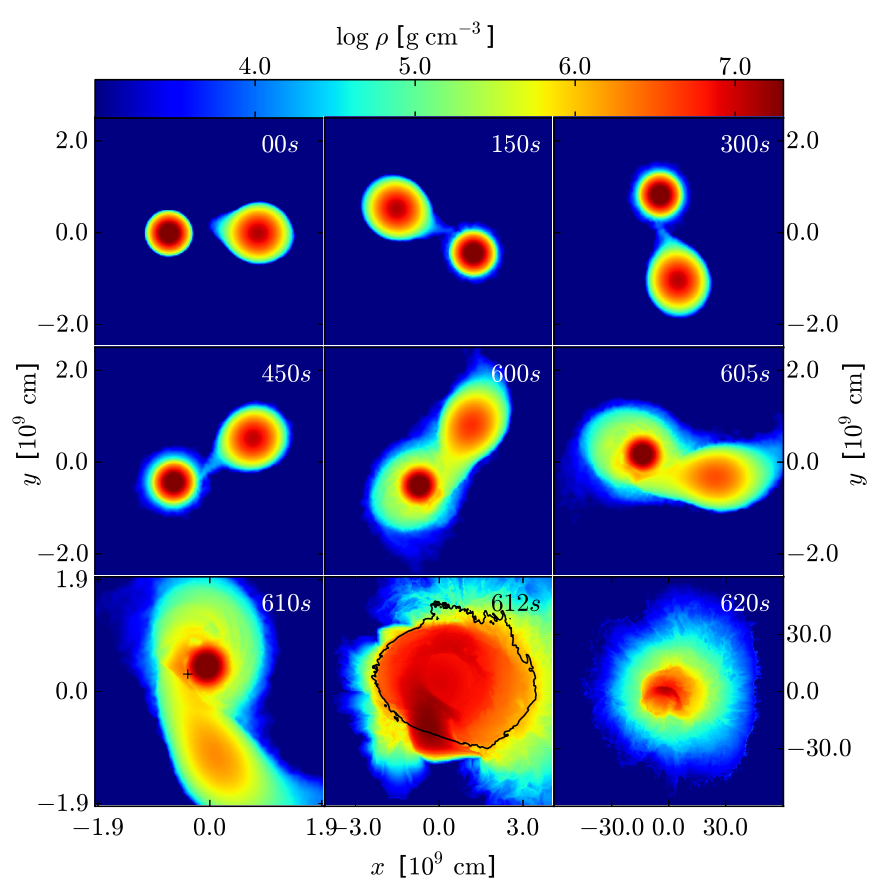

Figure 1. Snapshots of the merger of a $1.1 M_{\odot}$ and a $0.9 M_{\odot}$ carbon-oxygen white dwarf and the subsequent thermonuclear explosion. At the start of the simulation the binary system has an orbital period of $\approx 35 \mathrm{~s}$. The black cross indicates the position where the detonation is ignited. The black line shows the position of the detonation front. Color coded is the logarithm of the density. The last two panels have a different color scale ranging from $10^{-4} \mathrm{~g} \mathrm{~cm}^{-3}$ to $10^{6} \mathrm{~g} \mathrm{~cm}^{-3}$ and $10^{4} \mathrm{~g} \mathrm{~cm}^{-3}$, respectively.

(A color version of this figure is available in the online journal.)

\section{MERGER AND EXPLOSION}

The inspiral and merger is modeled using a modified version of the GADGET code (Springel 2005). Modifications include the Helmholtz equation of state (Timmes \& Swesty 2000) and a 13 isotope nuclear reaction network that contains all $\alpha$-elements from ${ }^{4} \mathrm{He}$ to ${ }^{56} \mathrm{Ni}$. Radiative cooling effects are not included in our simulation. A detailed description of the modifications will be given in a forthcoming paper. In addition, the maximum smoothing length of a particle was restricted to $10^{8} \mathrm{~cm}$. This affects only particles ejected from the binary system during the merger but leads to a significant speedup of the code. Since these particles are at very low densities and contain only less than $1 \%$ of the total mass, they have no noticeable influence on the explosion dynamics and observables.

The initial binary system consists of a $1.1 M_{\odot}$ and a $0.9 M_{\odot}$ carbon-oxygen white dwarf constructed from a total of $1.8 \times 10^{6}$ equal-mass particles. Both white dwarfs are set up in isolation and relaxed with an additional friction force for $100 \mathrm{~s}$. We then apply the method described in Dan et al. (2011) to slowly move the two white dwarfs close together. When the first particle of the less massive white dwarf crosses the inner Lagrange point, we stop and start the actual simulation. At this time, the binary system has an orbital period of about $35 \mathrm{~s}$.

The evolution of the binary system is shown in Figure 1. The mass transfer is stable for more than 15 orbits. After about $600 \mathrm{~s}$ the secondary white dwarf becomes dynamically unstable and is disrupted on a timescale of one orbit. As the material of the secondary is accreted violently onto the primary, material is compressed and heated up on the surface of the primary white dwarf. As a consequence hot spots form in which carbon burning is ignited. When the first hot spot reaches a temperature larger than $2.5 \times 10^{9} \mathrm{~K}$ at a density of about $2 \times 10^{6} \mathrm{~g} \mathrm{~cm}^{-3}$, we assume that a detonation forms (Seitenzahl et al. 2009). Note that despite the high resolution we use, we still tend to underestimate the temperature in the hot spot compared to even higher resolution simulations (Pakmor et al. 2011). Only future detailed investigations might be able to decide whether or not a detonation really forms but the conditions in our smoothed particle hydrodynamic simulations suggest that it is plausible.

At this time we map the whole state of the simulation on a uniform Cartesian grid with a resolution of $768^{3}$ grid cells and a box size of $4 \times 10^{9} \mathrm{~cm}$. About $0.03 M_{\odot}$ of material is lost by the mapping as it is outside the box. Since this material makes up less than $2 \%$ of the total mass and has a density too low to contribute significantly to nuclear burning (i.e., it stays unburned) it does not affect the dynamics of the ejecta or the synthetic observables derived from the model.

With these initial conditions we use the LEAFs code (Reinecke et al. 1999a) that applies the level-set technique to model detonation flames (Reinecke et al. 1999b; Fink et al. 2010). We ignite the detonation at the cell with the highest temperature and follow its propagation through the merged object until most of the material is burned. Figure 1 shows that the primary white dwarf and most parts of the secondary are already burned $2 \mathrm{~s}$ after the detonation formed. The energy release from nuclear burning unbinds the object. Using an expanding grid (Röpke 2005), we follow the dynamic ejecta until they reach homologous expansion about $100 \mathrm{~s}$ after the detonation was ignited. They have an asymptotic kinetic energy of $1.7 \times 10^{51} \mathrm{erg}$.

\section{NUCLEOSYNTHESIS}

In order to obtain detailed isotopic abundances of the ejecta, we add $10^{6}$ tracer particles to the simulation of the explosion that record their local temperature and density. In a post-processing step we run a detailed nuclear network containing 384 isotopes on these trajectories (Travaglio et al. 2004). To mimic the effect of solar metallicity, we choose the initial composition of the tracer particles as $47.5 \%{ }^{12} \mathrm{C}, 50 \%{ }^{16} \mathrm{O}$, and $2.5 \%{ }^{22} \mathrm{Ne}$ by mass.

In the explosion, a total of $0.7 M_{\odot}$ of iron-group elements are synthesized. They consist predominantly of radioactive ${ }^{56} \mathrm{Ni}$ $\left(0.61 M_{\odot}\right)$ with a small fraction of stable ${ }^{58} \mathrm{Ni}\left(0.03 M_{\odot}\right)$ and stable ${ }^{54} \mathrm{Fe}\left(0.02 M_{\odot}\right)$. In addition, $0.5 M_{\odot}$ of intermediate-mass elements are produced in the explosion. The ejecta contain about $0.5 M_{\odot}$ of oxygen and about $0.15 M_{\odot}$ of unburned carbon.

The spatial composition and density structure of the ejecta in homologous expansion are shown in Figure 2. Its inherently three-dimensional structure is a result of the state of the merged object at the time the detonation forms. Since the detonation propagates faster at higher densities, the primary white dwarf is burned first and its ashes expand. As they expand they sweep around the material of the partially intact secondary white dwarf that is still being burned. Therefore, the ashes of the primary have already expanded significantly before burning of the secondary is completed (roughly $1 \mathrm{~s}$ later), meaning that the ashes of the secondary completely dominate the center of the ejecta. Hence, when the ejecta reach the homologous expansion phase several $10 \mathrm{~s}$ later, the very inner parts of the ejecta do not contain material from the primary white dwarf and therefore no iron-group elements.

\section{COMPARISON WITH OBSERVATIONS}

Using the three-dimensional density structure from explosion modeling and the corresponding spatial abundance distribution 


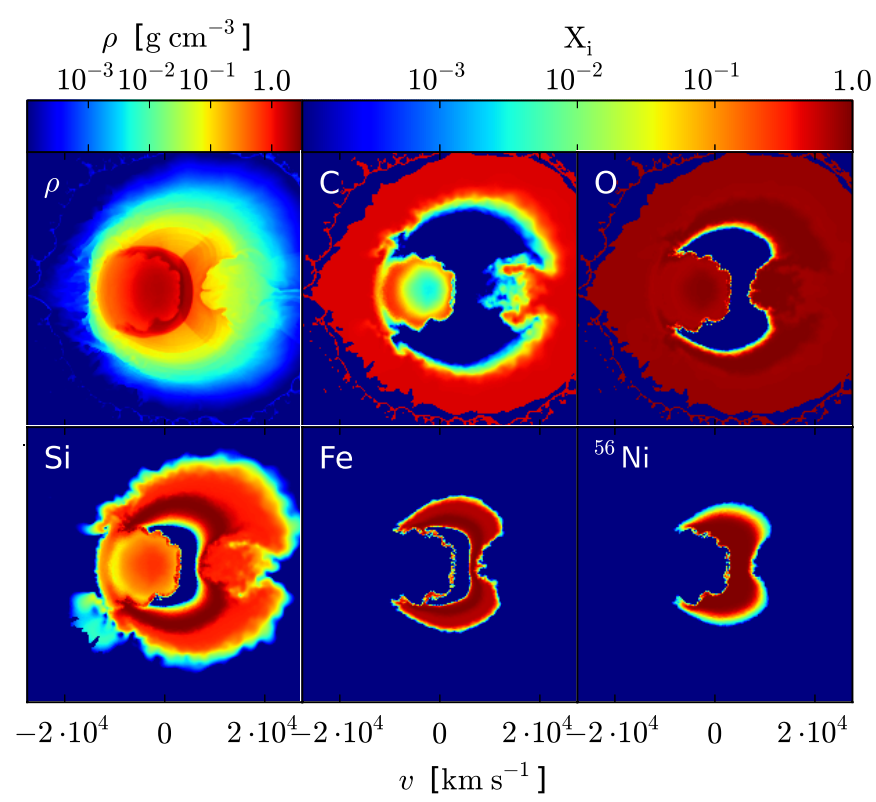

Figure 2. Density and composition of the final ejecta in homologous expansion $100 \mathrm{~s}$ after the explosion of a slice in the $x-z$ plane. The mass fraction is shown for carbon, oxygen, silicon, stable iron, and radioactive ${ }^{56} \mathrm{Ni}$.

(A color version of this figure is available in the online journal.)

from the tracer particles, we apply the Monte Carlo radiative transfer code ARTIS (Kromer \& Sim 2009; Sim 2007) to calculate synthetic spectra and light curves for our model.

Figure 3 shows angle-averaged and line-of-sight-dependent light curves of our model compared with several well-observed normal SNe Ia. The angle-averaged light curves show good agreement with observations in the optical bands and reproduce general properties of normal SNe Ia having a $B$-band rise time of 20.8 days, a peak brightness of $-19.6,-19.0$, and -19.2 in the $U, B$, and $V$ band, respectively, and a light curve decline rate in the $B$ band of $\Delta m_{15}=0.95$. These values are well within the range of normal SNe Ia (Hicken et al. 2009). More detailed values for selected bands of the angle-averaged light curves are given in Table 1.

In particular, the angle-averaged light curves in the $U$ and $R$ band agree very well with those observed for normal SNe Ia. The angle-averaged synthetic $B$-band light curve is about 0.3 mag fainter at maximum than the three supernovae we compare with and slightly brighter from 25 days after maximum onward. The angle-averaged $V$-band light curve of our model agrees well with the data up to about 30 days after maximum, but declines slightly more slowly afterward.

Due to the asymmetric ejecta structure, our model light curves show a non-negligible sensitivity to line-of-sight effects. In the $B$ band, for example, we find peak magnitudes between -19.5 and -18.7 . At the same time $\Delta m_{15}(B)$ varies between 0.5 and 1.4, roughly along the Phillips relation (Phillips et al. 1999). For the $U$ band, the scatter is even larger while it decreases in the redder bands. For lower photon energies, the asymmetry of the ejecta is less important, since the optical depths are smaller and photons probe a larger fraction of the total ejecta, thereby making the observables less sensitive to line-of-sight effects (compare Kromer \& Sim 2009; Kromer et al. 2010).

The angle-averaged light curve of our model in the $I$ band agrees well with the observations up to maximum light. Afterward it is between 0.2 and 0.7 mag brighter than our comparison
Table 1

Angle-averaged Light Curve Parameters for Selected Bands

\begin{tabular}{lccccc}
\hline \hline & Bolometric $^{\mathrm{a}}$ & $U$ & $B$ & $V$ & $R$ \\
\hline$t_{\max }$ & 18.6 & 18.0 & 20.8 & 23.2 & 21.6 \\
$\Delta m_{15}$ & 0.74 & 1.30 & 0.95 & 0.67 & 0.42 \\
$M\left(t_{\max }\right)$ & -19.2 & -19.6 & -19.0 & -19.2 & -19.2 \\
$M\left(t_{\max }(B)\right)$ & -19.1 & -19.5 & -19.0 & -19.2 & -19.2 \\
\hline
\end{tabular}


For comparability to observed bolometric light curves, the model light curve was reduced to UBVRIJHK bolometric.

SNe Ia. This offset (in the $I$ band after maximum) can be attributed to a flux excess in the Ca II NIR triplet in the synthetic spectra. This could point to an overabundance of calcium in the model. However, it is more likely to be a shortcoming of our radiative transfer treatment, which uses the simple van Regemorter approximation (van Regemorter 1962) to treat collisional excitation. Thus, we likely underestimate the effectiveness of cooling by forbidden transitions of heavy elements and therefore predict that too much cooling occurs via the strong dipole transitions of Ca II.

There is good agreement between model and data in the NIR bands $(J, H, K)$. In particular, our model reproduces time and brightness of the second peaks and even the light curve decline at more than 40 days after maximum quite accurately. However, high-precision modeling of the NIR light curves requires an extensive atomic data set to properly simulate flux redistribution by fluorescence (Kasen 2006; Kromer \& Sim 2009). Here, however, we have restricted ourselves for computational reasons to a simplified atomic data set (cd23_gf-5 of Kromer \& Sim 2009) with only $\sim 400,000$ lines. This has been shown to give reliable results in the optical bands but slightly underpredicts the NIR brightness (Kromer \& Sim 2009) between the primary and secondary peaks.

Figure 4 shows the angle-averaged spectrum of our model one day after $B$-band maximum. For comparison, we overplot an observed spectrum of the normal SN 2003du (Stanishev et al. 2007) at the same epoch. Our model shows most of the characteristic features of SNe Ia, particularly the defining Si II doublet at $\lambda \lambda 6347,6371$ but also the weaker Si II features at $\lambda \lambda 5958,5979$ and $\lambda \lambda 4128,4131$, the $\mathrm{Mg}$ II triplet at $\lambda 4481$ and the $\mathrm{Ca}$ II $\mathrm{H}$ and $\mathrm{K}$ absorption. The $\mathrm{S}$ II $\mathrm{W}$-feature at $\sim 5400 \AA$ (which is a blend of several lines) is also visible though it is weaker than in SN 2003du. In the red tail of the spectrum the Ca II NIR triplet is clearly visible as well as some indication of the O I triplet $\lambda \lambda 7772,7774,7775$. Moreover, the overall spectral shape and the velocity shifts of most of the line features in the observed spectrum are well reproduced, indicating that the velocity structure of our model is a good representation of that in real SNe Ia.

\section{DISCUSSION}

The dominant parameter that determines the brightness of the explosion in our model is the mass of the primary white dwarf. In the merger the primary remains mostly unaffected whereas the secondary white dwarf is destroyed. Therefore, because the density profile of the primary white dwarf to first order only depends on its mass and the density stays high enough to burn to iron-group elements $\left({ }^{56} \mathrm{Ni}\right)$ only in the primary white dwarf, the brightness of the supernova directly correlates with the mass of the primary white dwarf. 




Figure 3. Light curves of our model. The panels from top left to bottom right contain $U B V R I J H K$ bolometric and broadband $U, B, V, R, I, J, H, K$ light curves. The black line corresponds to the angle average of the model. Gray histograms show light curves along seven different lines-of-sight representative for the scatter caused by different (100) viewing angles including the most extreme light curves. The time is given relative to $B$-band maximum. The red symbols show observational data of three well-observed normal SNe Ia, SN 2001el (Krisciunas et al. 2003), SN 2003du (Stanishev et al. 2007), and SN 2005cf (Pastorello et al. 2007).

(A color version of this figure is available in the online journal.)

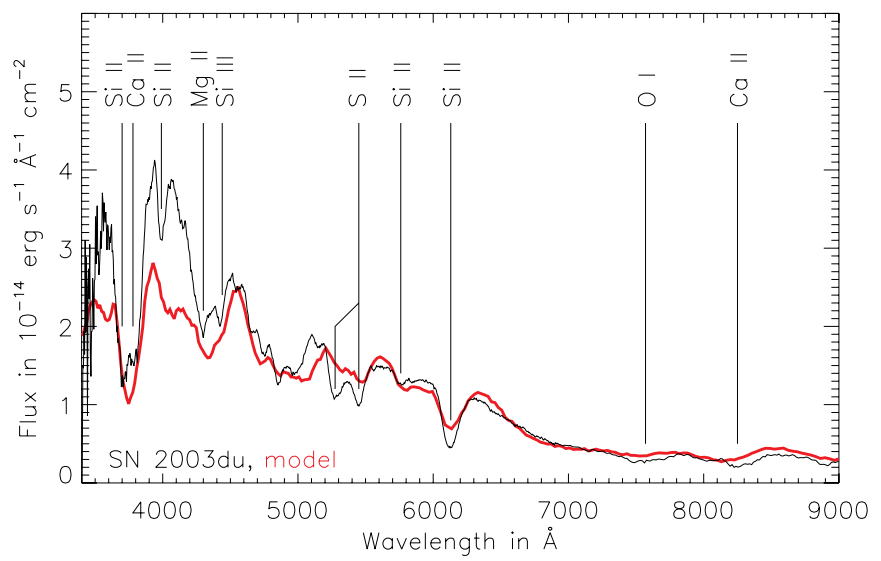

Figure 4. Maximum light spectrum of our model. The red line shows the spectrum of our model one day after maximum light in the $B$ band. The black line shows the observed spectrum of SN 2003du (Stanishev et al. 2007) at the same time.

(A color version of this figure is available in the online journal.)

Physical parameters of secondary importance are the mass of the secondary white dwarf and the composition of both white dwarfs $(\mathrm{C} / \mathrm{O}$ ratio and metallicity). The material of the secondary is burned only to intermediate-mass elements and oxygen and therefore only affects the brightness of the explosion indirectly. The metallicity of the two white dwarfs has only a small impact on the final composition of the ejecta by changing the mixture of iron-group elements synthesized (Sim et al. 2010).

The moment at which the detonation forms is an artificial parameter of our model. Physically, it will be determined by the properties of the binary system discussed above. Since we cannot resolve the formation of the detonation microscopically, however, we have to infer the most likely place and time for it to form from macroscopic properties. Once we assume that a detonation forms on the surface of the primary its exact place and time are only secondary effects: the choice for the moment of detonation affects the densities at which the material of the secondary white dwarf is burned (since the detonation occurs while the secondary is being destroyed) but it does not strongly affect the burning of the primary because it is not dynamically affected by the merger.

Compared to the merger of two $0.9 M_{\odot}$ white dwarfs (Pakmor et al. 2010) the merger we present here mainly differs in the mass of the primary white dwarf. Since it is considerably more massive, its central density is higher and its material is burned predominantly to iron-group elements. In addition, the mass ratio of the binary system we model here is well below unity. Therefore, the more compact primary is not subject to noticeable tidal forces from the secondary. 
In addition, although exact initial conditions may weaken the merger, we show that it still leads to the formation of hot spots on the surface of the primary, in which densities and temperatures are sufficiently high that a detonation is plausible. This is consistent with the properties of hot spots seen in previous studies with less relaxed initial conditions (Pakmor et al. 2010, 2011) and also with very recent simulations by Raskin et al. (2012) who found similar hot spots in calculations with relaxed initial conditions and only slightly smaller resolution than our simulation. Note that Dan et al. (2012) do not find hot spots in similar systems with exact initial conditions but more than a factor of 10 fewer particles.

Our model can be interpreted as a pure detonation of an isolated sub-Chandrasekhar mass white dwarf as described in Sim et al. (2010) with an additional component from the remains of the secondary white dwarf surrounding the system. Quantitatively, however, the color light curves of our model agree significantly better with observations than the toy models of Sim et al. (2010). In particular, our model also shows good agreement in the NIR bands and reproduces the position of the secondary peaks in these bands very well.

This difference to the toy models is likely to be associated with a different density profile and composition structure in the inner parts of the ejecta. As discussed in Section 3 this is a result of the presence of the secondary white dwarf and its interaction with ashes of the primary when they expand. Also, the asymptotic kinetic energy per mass of the ejecta is smaller for our merger model than for detonations of isolated sub-Chandrasekhar mass white dwarfs, because the burning of the secondary white dwarf is less complete and hence the average energy release per mass is smaller.

Overall, our explosion reproduces observational data of normal SNe Ia of the same brightness reasonably well. Since the time span from the onset of mass transfer between the two white dwarfs until the ejecta reach homologous expansion is very short (only of the order of a few minutes) there is no extended outer envelope the explosion ejecta interact with later than a few minutes after the explosion, in contrast to the model by Fryer et al. (2010).

Observational constraints (Napiwotzki et al. 2005, 2007) on the number of double white dwarf binaries significantly more massive than a Chandrasekhar mass are still not very restrictive. If violent mergers contribute noticeably to the total SNe Ia rate, our calculations suggest that it will be hard to distinguish the merger events from the bulk of normal SNe Ia via optical/NIR light curves and optical spectra. Further detailed work is therefore needed to investigate whether there are other characteristic properties of SN Ia explosions from violent white dwarf mergers and to explore how the observational display changes with the properties of their progenitor system.

This work was supported by the Deutsche Forschungsgemeinschaft via the Transregional Collaborative Research Center
TRR 33 "The Dark Universe," the Excellence Cluster EXC153 "Origin and Structure of the Universe" and the Emmy Noether Program (RO/3676 1-1). Parts of the simulations were carried out at the John von Neumann Institute for Computing (NIC) in Jülich, Germany (project hmu14). R.P. gratefully acknowledges financial support of the Klaus Tschira Foundation.

\section{REFERENCES}

Badenes, C., Hughes, J. P., Bravo, E., \& Langer, N. 2007, ApJ, 662, 472 Bloom, J. S., Kasen, D., Shen, K. J., et al. 2012, ApJ, 744, 17

Dan, M., Rosswog, S., Guillochon, J., \& Ramirez-Ruiz, E. 2011, ApJ, 737, 89 Dan, M., Rosswog, S., Guillochon, J., \& Ramirez-Ruiz, E. 2012, arXiv:1201.2406

Fink, M., Hillebrandt, W., \& Röpke, F. K. 2007, A\&A, 476, 1133

Fink, M., Röpke, F. K., Hillebrandt, W., et al. 2010, A\&A, 514, A53

Fryer, C. L., Ruiter, A. J., Belczynski, K., et al. 2010, ApJ, 725, 296

Gilfanov, M., \& Bogdán, Á. 2010, Nature, 463, 924

Hancock, P. P., Gaensler, B. M., \& Murphy, T. 2011, ApJ, 735, L35

Hicken, M., Challis, P., Jha, S., et al. 2009, ApJ, 700, 331

Howell, D. A. 2011, Nature Commun., 2, 350

Iben, I., Jr., \& Tutukov, A. V. 1984, ApJS, 54, 335

Kasen, D. 2006, ApJ, 649, 939

Krisciunas, K., Suntzeff, N. B., Candia, P., et al. 2003, AJ, 125, 166

Kromer, M., \& Sim, S. A. 2009, MNRAS, 398, 1809

Kromer, M., Sim, S. A., Fink, M., et al. 2010, ApJ, 719, 1067

Leonard, D. C. 2007, ApJ, 670, 1275

Maoz, D., Sharon, K., \& Gal-Yam, A. 2010, ApJ, 722, 1879

Napiwotzki, R., Karl, C. A., Nelemans, G., et al. 2005, in ASP Conf. Ser. 334 14th European Workshop on White Dwarfs, ed. D. Koester \& S. Moehler (San Francisco, CA: ASP), 375

Napiwotzki, R., Karl, C. A., Nelemans, G., et al. 2007, in ASP Conf. Ser. 372, 15th European Workshop on White Dwarfs, ed. R. Napiwotzki \& M. R. Burleigh (San Francisco, CA: ASP), 387

Nomoto, K., \& Iben, I., Jr. 1985, ApJ, 297, 531

Nomoto, K., \& Kondo, Y. 1991, ApJ, 367, L19

Pakmor, R., Hachinger, S., Röpke, F. K., \& Hillebrandt, W. 2011, A\&A, 528, A117

Pakmor, R., Kromer, M., Röpke, F. K., et al. 2010, Nature, 463, 61

Pastorello, A., Taubenberger, S., Elias-Rosa, N., et al. 2007, MNRAS, 376, 1301

Phillips, M. M., Lira, P., Suntzeff, N. B., et al. 1999, AJ, 118, 1766

Raskin, C., Scannapieco, E., Fryer, C., et al. 2012, ApJ, 746, 62

Reinecke, M., Hillebrandt, W., \& Niemeyer, J. C. 1999a, A\&A, 347, 739

Reinecke, M., Hillebrandt, W., Niemeyer, J. C., Klein, R., \& Gröbl, A. 1999b, A\&A, 347, 724

Röpke, F. K. 2005, A\&A, 432, 969

Ruiter, A. J., Belczynski, K., \& Fryer, C. 2009, ApJ, 699, 2026

Saio, H., \& Nomoto, K. 1998, ApJ, 500, 388

Seitenzahl, I. R., Meakin, C. A., Townsley, D. M., Lamb, D. Q., \& Truran, J. W. 2009, ApJ, 696, 515

Sim, S. A. 2007, MNRAS, 375, 154

Sim, S. A., Röpke, F. K., Hillebrandt, W., et al. 2010, ApJ, 714, L52

Springel, V. 2005, MNRAS, 364, 1105

Stanishev, V., Goobar, A., Benetti, S., et al. 2007, A\&A, 469, 645

Timmes, F. X., \& Swesty, F. D. 2000, ApJS, 126, 501

Travaglio, C., Hillebrandt, W., Reinecke, M., \& Thielemann, F.-K. 2004, A\&A, 425, 1029

van Regemorter, H. 1962, ApJ, 136, 906

Webbink, R. F. 1984, ApJ, 277, 355

Whelan, J., \& Iben, I. J. 1973, ApJ, 186, 1007

Yoon, S.-C., Podsiadlowski, P., \& Rosswog, S. 2007, MNRAS, 380, 933 